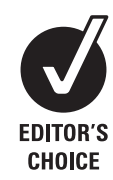

Department of

Gastroenterology, Affiliated Hospital of Luzhou Medical College, Sichuan, China

Correspondence to Dr Xiangsheng Fu, Department of Gastroenterology, Affiliated Hospital of Luzhou Medical College, Taiping Street 25\# Region Jiangyang, Luzhou, Sichuan 646000, China; drfuxs@gmail.com

Accepted 2 February 2012

\title{
Wnt signalling pathway in the serrated neoplastic pathway of the colorectum: possible roles and epigenetic regulatory mechanisms
}

\author{
Xiangsheng Fu, Li Li, Yan Peng
}

\begin{abstract}
The role of Wnt signalling in the serrated neoplastic pathway of colorectal tumourigenesis appears to be heterogeneous. Wnt pathway abnormalities contribute to the progression of at least a subset of traditional serrated adenomas of the colorectum, but may play a less active role in its pathogenesis compared with that in conventional adenoma-carcinoma. However, immunohistochemical studies of $\beta$-catenin in sessile serrated adenomas have shown wide variability, producing conflicting results on Wnt signalling activation in sessile serrated adenomas. DNA methylation, involving APC, SFRPs and mutated in colorectal cancer (MCC), may bridge the mutational gap of $A P C$ or $\beta$-catenin for activating Wnt signalling in serrated adenomas of the colorectum.
\end{abstract}

\section{INTRODUCTION}

Most colorectal cancers (CRCs) develop through a conventional adenoma (CA)-carcinoma sequence. ${ }^{12}$ However, recent increasing evidence suggests that $10 \%-20 \%$ of CRCs develop through the "serrated polyp-neoplasia pathway'. ${ }^{3-6}$ The recognition of this pathway in recent years has led to a paradigm shift in our understanding of the molecular basis of CRC and significant changes in clinical practice. ${ }^{7}$

Serrated polyps are histologically classified into hyperplastic polyps (HPs), traditional serrated adenomas (TSAs) and sessile serrated adenomas (SSAs). ${ }^{8}{ }^{9}$ HPs are characterised by their small size (usually $<0.5 \mathrm{~cm}$ ), a predominantly left-sided location and generally regarded as non-neoplastic. Histologically, HPs have prominent serrations towards the luminal surface and crypts that narrow towards the muscularis mucosae (figure 1A). HPs are further classified as microvesicular $\mathrm{HP}$, goblet cell HP, and mucin-depleted HP. ${ }^{10}$ Some data suggest that MVHP (especially right-sided) may be a precursor to more advanced SSAs. ${ }^{8} 1112$

TSAs were first described by Longacre and Fenoglio-Preiser ${ }^{13}$ in 1990 as serrated adenomas (SAs) that exhibit cytological dysplasia reminiscent of classical adenomas and a serrated architecture resembling HPs. TSAs are characterised by a predilection for the distal colon and rectum, apparent dysplasia, nuclear stratification and penicillate nuclei with abundant to moderate eosinophilic cytoplasm (figure 1B). Although TSAs were considered to be lesions of minor importance because of its low prevalence $(2 \%-3.5 \%),{ }^{7}$ recent reports claimed that TSAs have a higher growth rate than CAs, and that the subsequent cancer risk rate at least equals that of CAs. ${ }^{14} 15$
In 1996, the term sessile serrated adenoma was introduced to refer to a subset of serrated lesions that cytologically resemble HPs but is distinguished from HPs on the basis of crypt dilation, branching and horizontal spreading (figure 1C, D). ${ }^{16}$ Unlike HPs, SSAs are characterised by a larger size, a predominantly right-sided location and the neoplastic potential. However, histopathological diagnosis of SSAs may be difficult, as they closely resemble HPs. ${ }^{17} 18$ The concept of SSAs was reintroduced in 2003 and were accounted to represent $18 \%-22 \%$ of serrated polyps. ${ }^{8} 19$ With the growing evidence of malignant change of SSA in epidemiological backgrounds, SSA has been considered to be the precursor of some microsatellite instability (MSI)high carcinomas of the proximal colon. ' Some researchers concluded that SSAs were high-risk lesions, with $15 \%$ of the SSA patients developing subsequent CRCs or adenomas with high-grade dysplasia. ${ }^{20}$ Furthermore, there is evidence that the neoplastic progression within this pathway is faster than within the classical adenomacarcinoma sequence. ${ }^{14}{ }^{21}$ However, the details of the molecular mechanism of stepwise progression from SSA to early invasive carcinoma remain unclear.

It is well known that Wnt signalling pathway involving $\beta$-catenin plays a critical role in colorectal carcinogenesis (figure 2). ${ }^{22}{ }^{23} \beta$-Catenin and its regulators, such as adenomatous polyposis coli (APC), is well studied in CAs and related carcinomas. However, the published data on Wnt pathway activation in SAs (including TSAs and SSAs) are controversial. ${ }^{24-28}$

The purpose of this review is to discuss the possible role and regulatory mechanisms of Wnt signalling pathway in the development and progression of serrated polyps.

\section{WNT SIGNALLING PATHWAY MAY PLAY A LESS ACTIVE ROLE IN THE PATHOGENESIS OF A SUBSET OF TSAS}

Activation of the Wnt signalling pathway in CRC occurs almost invariably through mutation of the two key molecules: APC and $\beta$-catenin (CTNNB1). ${ }^{29}$ Up to $80 \%$ of CRCs have $A P C$ mutations, whereas $10 \%$ of CRCs have $\beta$-catenin mutations. ${ }^{29} \mathrm{An}$ important function of the APC protein is to form a complex with the key effector $\beta$-catenin. Loss of APC function leads to translocation of $\beta$-catenin from the lateral cell membrane to the nucleus, where it promotes the transcription of multiple genes involved in tumour growth and invasion. ${ }^{30}$ 
Figure 1 Histological features of serrated polyps. (A) Hyperplastic polyp: The serrations of the epithelium are confined to the upper half of the crypts, and the numbers of goblet cells are decreased relative to the normal mucosa. (B) Traditional serrated adenoma: This exophytic polyp shows serrated epithelial changes in association with cytoplasmic eosinophilia, nuclear enlargement and crowding. $(C, D)$ Sessile serrated adenoma: In contrast to the hyperplastic polyp shown in $A$, the architecture of the crypts is distorted, seen as basal crypt dilatation (arrows) and crypt branching. H\&E staining.
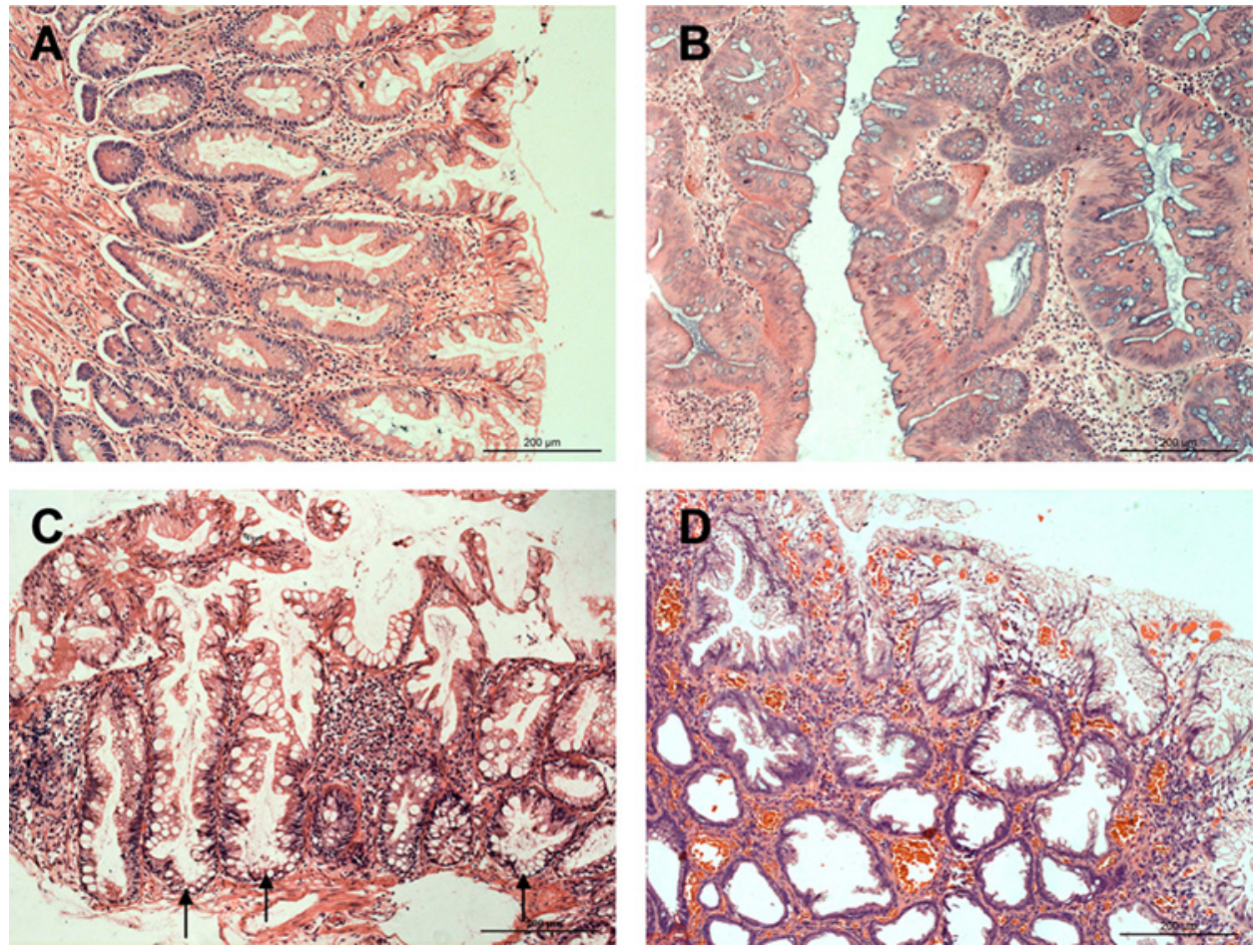

Although aberrant activation of Wnt signalling is a prerequisite for the initiation of most $\mathrm{CRCs},{ }^{29}$ its role in TSAs is still controversial.

Some studies report a high incidence of APC gene mutation/ loss of heterozygosity (LOH) in TSAs. Hiyama and colleagues ${ }^{25}$ demonstrated APC mutation in $40 \%(2 / 5)$ of TSAs. In Matsumoto's study, ${ }^{31} 3 / 3$ TSAs with familial adenomatous polyposis had germline $A P C$ mutation. In another study, $\mathrm{LOH}$ of $A P C$ was seen in $20 \%(7 / 35)$ of TSAs. ${ }^{32}$ However, most of these studies were limited by their small sample sizes.

Not all molecular studies have shown consistent findings. Sawyer et $a l^{33}$ reported that APC mutations occurred in $12.8 \%$

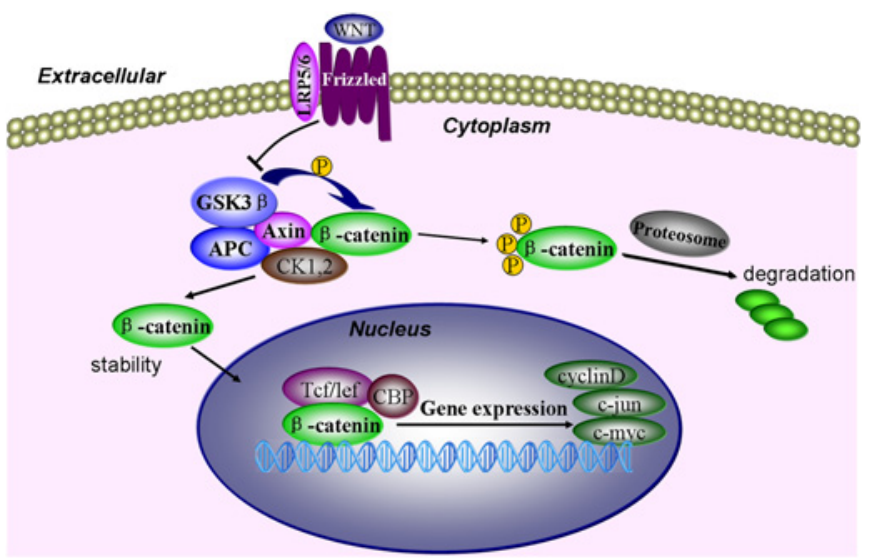

Figure 2 A canonical Wnt signalling pathway. In the absence of Wnt signal, $\beta$-catenin is recruited into the APC/Axin/GSK3 $\beta /$ CK destruction complex and phosphorylated by GSK3 $\beta$. The hyperphosphorylated $\beta$ catenin is targeted for degradation by the proteosome. Binding of Wnt ligand to a Frizzled/LRP-5/6 receptor complex, leading to the inhibition of APC/Axin/GSK3 $\beta /$ CK-mediated $\beta$-catenin degradation. Stabilised $\beta$ catenin forms a transcriptional complex with Tcf/lef and activates downstream targets such as c-myc.
(5/39) of TSAs. In a study by Dehari et al, ${ }^{27}$ APC mutations were detected in $3.8 \%(1 / 26)$ of TSAs, and in none of the four adenocarcinomas with TSAs, but were detected in $66.7 \%(8 / 12)$ of tubular adenomas and in $50 \%$ (4/8) of adenocarcinomas with tubular adenomas. Uchida and colleagues ${ }^{26}$ found that $37.0 \%$ $(10 / 27)$ of CAs showed somatic mutations of APC but no APC mutation was identified in 12 TSAs. In our previous study, ${ }^{34}$ the frequency of $A P C$ gene mutation in TSAs $(8.3 \%, 1 / 12)$ was significantly lower than that in CAs $(56.3 \%, 9 / 16)$ and CRCs $(50.0 \%, 7 / 14)$. These studies looking for APC mutations in TSAs have shown a low rate of detection, relative to the $50 \%-63 \%$ reported in CAs. ${ }^{35}$

Sawyer et $a l^{33}$ argued that APC mutations in these studies were likely to be underestimated because of poor quality of DNA retrieved from the archival material. However, we noticed that most of these studies had DNA from paraffin tissue sections of CAs as controls, which showed a high frequency of $A P C$ mutations. Moreover, even 3/3 TSAs had germline APC mutations in the study by Matsumoto et al, ${ }^{31}$ these mutations had been shown to cluster at the proximal exons 9 and at the distal $3^{\prime}$ end of exon 15. It is reported that these mutations do not significantly affect the tumour-suppressor function of APC, ${ }^{36} 37$ suggesting that TSA of familial adenomatous polyposis may be a phenotype associated with less impaired APC function, although this study was limited by its very small sample size. Indeed, our previous study found that expression of APC protein in TSAs was higher than that in CAs, although the difference was not statistically significant. ${ }^{34}$ These findings suggest that APC's function in TSAs is less likely to be impaired compared with that in CA-carcinoma sequence.

Previously published results of $\beta$-catenin expression in TSAs had shown mixed findings. For instance, in a study by Jiao et $a l^{24}$ in 2008, nuclear $\beta$-catenin positive cells were found in all TSAs (43 cases), but there was no significant difference in nuclear $\beta$-catenin positive rates between TSAs $(30 \%)$ and CAs $(30 \%){ }^{24}$ In another study, $\mathrm{Oh}$ et $a^{38}$ also noted a relatively high frequency of positivity for $\beta$-catenin in TSAs $(61 \%, 14 / 23)$. 
However, contradictory results have been reported by other investigators. Sawyer et $a^{33}$ evaluated $\beta$-catenin expression in 39 TSAs and found that only $12.8 \%(5 / 39)$ of TSAs showed nuclear $\beta$-catenin staining and $1 / 39$ showed $\beta$-catenin mutation. Yamamoto et $a l^{28}$ reported that widespread or focal nuclear $\beta$-catenin expression was demonstrated in $7 \%$ of TSAs $(3 / 45)$, relative to $61 \%$ of CAs (43/71). Cytoplasmic immunostaining for $\beta$-catenin was demonstrated in $16 \%$ of TSAs $(7 / 45)$ relative to $77 \%$ of CAs $(55 / 71)$. No mutation in exon 3 of $\beta$-catenin was found in TSAs, while $7 \%$ of CAs $(5 / 71)$ had $\beta$-catenin mutations. On the basis of these results, Yamamoto et $a l^{28}$ concluded that intracellular localisation of $\beta$-catenin may not be associated with an early event of the tumour progression in most TSAs. However, Yachida et $a^{39}$ reviewed the immunostaining results presented in that paper, and argued that the figure indicated scattered positive nuclear labelling (not negative staining, as the authors pointed out).

In our previous study in $2009,{ }^{34}$ nuclear expression of $\beta$-catenin was not seen in any of the TSAs (12 cases). Cytoplasmic accumulation of $\beta$-catenin was demonstrated in $41.7 \%(5 / 12)$ of TSAs, relative to $68.6 \%(11 / 16)$ of CAs. No $\beta$-catenin mutation was detected in any of the 12 TSAs. In our more recent study in $2011,{ }^{40}$ nuclear labelling of $\beta$-catenin was detected in $19.6 \%$ of CAs (51 cases), but in none of the TSAs (37cases). Cytoplasmic accumulation of $\beta$-catenin was found in $21.6 \%$ of TSAs, significantly lower than that in CAs $(60.8 \%)$.

Although conflicting results exist, Wnt pathway abnormalities (involving changes in APC/ $\beta$-catenin) may contribute to the progression of at least a subset of TSAs of the colorectum. However, it is worth noting that there seems to be a trend that frequency of Wnt signalling abnormality in TSAs is lower than that in CAs, raising the possibility that the Wnt pathway may play a less active role in the pathogenesis of a certain proportion of TSAs.

\section{CONFLICTING RESULTS ON ACTIVATION OF THE WNT SIGNALLING PATHWAY IN SSAS}

The published data on Wnt pathway activation in SSAs are more conflicting. In a study by Wu et $a l^{41}$ the authors surprisingly found that predominant nuclear labelling for $\beta$-catenin was noted in 9/22 SSAs (41\%) but in none of the HPs. In all SSAs with nuclear labelling, membranous labelling was significantly decreased. They expanded upon this observation of $\beta$-catenin expression in a larger set of serrated polyps of the colorectum. ${ }^{39}$ Immunolabelling for $\beta$-catenin confirmed the presence of nuclear accumulation in 35/54 (67\%) SSAs, compared with 0/12 HPs. Furthermore, nuclear $\beta$-catenin labelling was seen in 8/27 (29\%) SSAs without dysplasia, but in $27 / 27(100 \%)$ SSAs with dysplasia. Molecular study revealed that aberrant nuclear labelling of $\beta$-catenin occurred in a background of BRAF activation. ${ }^{39}$ Their findings suggest that aberrant Wnt signalling has a role in the development of a subset of SSAs and nuclear accumulation of $\beta$-catenin may be closely related to the early progression of SSAs.

The results from Sandmeier et al ${ }^{42}$ showed that abnormal $\beta$-catenin expression (loss of membranous $\beta$-catenin expression and nuclear $\beta$-catenin accumulation) was present in $37.5 \%(6 / 16)$ of SSAs, but not significantly different from $50.0 \%(6 / 12)$ of HPs. By contrast, in a study by Joo et al, ${ }^{43}$ nuclear $\beta$-catenin staining was observed in $0 \%$ of SSAs. However, this study was limited by its small sample size $(n=10)$. In another study by Fujita et al, ${ }^{44}$ no nuclear $\beta$-catenin expression was seen in 53 SSAs, although nuclear accumulation of $\beta$-catenin was observed in $50 \%(6 / 12)$ of
SSAs with neoplastic progression (SSANs, including intraepithelial high-grade dysplasia and submucosal invasive carcinoma). These results are consistent with our previous study showing that nuclear labelling of $\beta$-catenin was detected in none of the SSAs and HPs tested. ${ }^{40}$ These results indicate that Wnt pathway activation, seen as nuclear labelling for $\beta$-catenin, is unlikely to contribute to tumourigenesis in most SSAs.

Remarkably, these immunohistochemical studies of $\beta$-catenin in SSAs have shown wide variability in terms of proportion with nuclear staining, from $0 \%$ to $100 \%$. Many reasons may contribute to these differing results, including intrinsic tumour heterogeneity, different stages of adenomas, small sample size, differences in antigen retrieval, antibodies and staining procedures used by each laboratory and different evaluations of immunostaining with varying degrees of sensitivity. Especially, conclusive studies of the Wnt signalling pathway in SSAs have been limited by the heterogeneity of SSAs classified in this category. Diagnostic criteria and nomenclature for these lesions are inconsistent and partly confusing. ${ }^{18} 45$ Therefore, some SSAs are still misdiagnosed and wrongly categorised, which may lead to differing results. Fortunately, a standardised diagnostic nomenclature of different serrated polyps was formulated in $2010,{ }^{45}$ meriting future research and clinical management of serrated polyps.

\section{EPIGENETIC MECHANISMS IN ACTIVATING WNT SIGNALLING OF THE SERRATED NEOPLASTIC PATHWAY}

Activating mutations in CTNNB1 and APC are a common feature of CA-carcinoma sequence. Up to $80 \%$ of CRCs have APC mutations, whereas $10 \%$ of CRCs have $\beta$-catenin mutations. ${ }^{29}$ However, most studies reported infrequent APC mutations in TSAs compared with that in CAs. ${ }^{26} 2733{ }^{34} \mathrm{MSI}$ is classically a feature of TSAs that appear to distinguish them from CAs. ${ }^{33} 4647$ It is reported that activating mutations in CTNNB1, not APC genetic inactivation, are frequent features of MSI CRCs that similarly cause stabilisation of $\beta$-catenin. ${ }^{48} 49$ However, activating mutations in CTNNB1 is seldom seen in TSAs. $^{28} 3334$ Yachida and colleagues ${ }^{39}$ also found no mutations in CTNNB1 as a mechanism for Wnt activation, although they noted frequent $\beta$-catenin nuclear labelling in SSAs.

These results suggest that nuclear accumulation of $\beta$-catenin may also occur in the absence of mutations in the $A P C / \beta$-catenin pathway. ${ }^{33}$ Previous studies have revealed that DNA methylation could provide an alternative mechanism to gene mutation for silencing $A P C .^{50-52}$ Moreover, Suzuki et al ${ }^{53}$ provided compelling evidence of the Wnt pathway activation in CRC cell lines by hypermethylation of the Wnt pathway antagonists SFRP1, SFRP2 and SFRP5. Indeed, a high frequency of DNA methylation is common in SAs and may play an important role in their pathogenesis. ${ }^{3}{ }^{54-56}$ Thus, it is conceivable that DNA methylation could bridge the mutational gap of $A P C$ or CTNNB1 for activating Wnt signalling in SAs. ${ }^{39}$ Dhir et al ${ }^{57}$ reported that SFRPS (1, 2, 4 and 5) were methylated frequently in SSAs and SSAs with dysplasia, but infrequently in HPs, providing evidence that the Wnt signalling pathway may be activated by epigenetic mechanisms in SSAs. Our previous study has also shown that hypermethylation of $A P C$ promoter $1 \mathrm{~A}$, instead of mutations involving $A P C$ and $\beta$-catenin, contributed to moderate activation of Wnt signalling in a subset of TSAs. ${ }^{34}$ Whether APC methylation also plays a role in tumourigenesis of SSA remains to be tested.

Recent studies have shown that mutated in colorectal cancer (MCC), a candidate tumour suppressor, can also interact with 


\section{Take-home messages}

- Wnt pathway abnormalities contribute to the progression of a subset of TSAs, but may play a less active role compared to that in conventional adenoma-carcinoma sequence.

- The role of Wnt signaling in the progression of SSA is still controversial. Further studies are needed to clarify this issue.

- DNA methylation may bridge the mutational gap for activating Wnt signaling in the "serrated polyp-neoplasia pathway" of the colorectum.

$\beta$-catenin, block $\beta$-catenin/TCF/LEF DNA binding, and affect endogenous $\beta$-catenin localisation/levels. ${ }^{58}$ Moreover, MCC methylation, associated with BRAF mutation, was more common in serrated polyps than in CAs. ${ }^{59}$ Therefore, MCC inactivation by promoter methylation could be an initiating Wnt pathway-activating event in the serrated neoplasia pathway. Additional studies will be required to address this possibility.

Contributors XF drafted the paper. LL revised the manuscript. YP edited the paper. All three authors take responsibility for the content of the paper.

Competing interests None.

Provenance and peer review Not commissioned; externally peer reviewed.

\section{REFERENCES}

1. Morson B. President's address. The polyp-cancer sequence in the large bowel. Proc R Soc Med 1974;67:451-7.

2. Hill MJ, Morson BC, Bussey HJ. Aetiology of adenoma-carcinoma sequence in large bowel. Lancet 1978;1:245-7.

3. Grady WM. CIMP and colon cancer gets more complicated. Gut 2007:56:1498-500.

4. Goldstein NS. Serrated pathway and APC (conventional)-type colorectal polyps: molecular-morphologic correlations, genetic pathways, and implications for classification. Am J Clin Pathol 2006;125:146-53.

5. Jass JR. Serrated route to colorectal cancer: back street or super highway? J Pathol 2001;193:283-5.

6. Makinen MJ. Colorectal serrated adenocarcinoma. Histopathology 2007:50:131-50.

7. Leggett B, Whitehall V. Role of the serrated pathway in colorectal cancer pathogenesis. Gastroenterology 2010;138:2088-100.

8. Torlakovic E, Skovlund E, Snover DC, et al. Morphologic reappraisal of serrated colorectal polyps. Am J Surg Pathol 2003;27:65-81.

9. Snover DC, Jass JR, Fenoglio-Preiser C, et al. Serrated polyps of the large intestine: a morphologic and molecular review of an evolving concept. Am J Clin Pathol 2005; 124:380-91.

10. Lauwers GY, Chung DC. The serrated polyp comes of age. Gastroenterology 2006;131:1631-4

11. Kim KM, Lee EJ, Ha S, et al. Molecular features of colorectal hyperplastic polyps and sessile serrated adenoma/polyps from Korea. Am J Surg Pathol 2011;35:1274-86

12. Snover DC. Update on the serrated pathway to colorectal carcinoma. Hum Pathol 2011:42:1-10.

13. Longacre TA, Fenoglio-Preiser CM. Mixed hyperplastic adenomatous polyps/ serrated adenomas. A distinct form of colorectal neoplasia. Am J Surg Pathol 1990:14:524-37.

14. Lazarus R, Junttila $\mathrm{OE}$, Karttunen $\mathrm{TJ}$, et al. The risk of metachronous neoplasia in patients with serrated adenoma. Am J Clin Pathol 2005;123:349-59.

15. Chandra A, Sheikh AA, Cerar A, et al. Clinico-pathological aspects of colorectal serrated adenomas. World J Gastroenterol 2006;12:2770-2.

16. Cunningham KS, Riddell RH. Serrated mucosal lesions of the colorectum. Curr Opin Gastroenterol 2006:22:48-53.

17. Farris AB, Misdraji J, Srivastava A, et al. Sessile serrated adenoma: challenging discrimination from other serrated colonic polyps. Am J Surg Pathol 2008;32:30-5.

18. Torlakovic EE, Gomez JD, Driman DK, et al. Sessile serrated adenoma (SSA) vs. traditional serrated adenoma (TSA). Am J Surg Pathol 2008;32:21-9.

19. Goldstein NS, Bhanot $P$, Odish E, et al. Hyperplastic-like colon polyps that preceded microsatellite-unstable adenocarcinomas. Am J Clin Pathol 2003;119:778-96.

20. Lu FI, van Niekerk de W, Owen D, et al. Longitudinal outcome study of sessile serrated adenomas of the colorectum: an increased risk for subsequent right-sided colorectal carcinoma. Am J Surg Pathol 2010;34:927-34.
21. Goldstein NS. Small colonic microsatellite unstable adenocarcinomas and highgrade epithelial dysplasias in sessile serrated adenoma polypectomy specimens: a study of eight cases. Am J Clin Pathol 2006;125:132-45.

22. Bienz $\mathbf{M}$, Clevers $\mathrm{H}$. Linking colorectal cancer to Wnt signaling. Cell 2000; 103:311-20.

23. Sena P, Saviano M, Monni S, et al. Subcellular localization of beta-catenin and APC proteins in colorectal preneoplastic and neoplastic lesions. Cancer Lett 2006;241:203-12.

24. Jiao YF, Nakamura S, Sugai T, et al. Serrated adenoma of the colorectum undergoes a proliferation versus differentiation process: new conceptual interpretation of morphogenesis. Oncology 2008;74:127-34.

25. Hiyama T, Yokozaki H, Shimamoto F, et al. Frequent p53 gene mutations in serrated adenomas of the colorectum. J Pathol 1998;186:131-9.

26. Uchida $\mathbf{H}$, Ando $\mathrm{H}$, Maruyama $\mathrm{K}$, et al. Genetic alterations of mixed hyperplastic adenomatous polyps in the colon and rectum. Jpn J Cancer Res 1998;89: 299-306.

27. Dehari R. Infrequent APC mutations in serrated adenoma. Tohoku J Exp Med 2001:193:181-6.

28. Yamamoto T, Konishi $K$, Yamochi T, et al. No major tumorigenic role for betacatenin in serrated as opposed to conventional colorectal adenomas. $\mathrm{Br} \mathrm{J}$ Cancer 2003;89:152-7.

29. Schneikert J, Behrens J. The canonical Wnt signalling pathway and its APC partne in colon cancer development. Gut 2007:56:417-25.

30. Korinek V, Barker N, Morin PJ, et al. Constitutive transcriptional activation by a beta-catenin-Tcf complex in APC-/- colon carcinoma. Science 1997; 275:1784-7

31. Matsumoto T, lida M, Kobori $Y$, et al. Serrated adenoma in familial adenomatous polyposis: relation to germline APC gene mutation. Gut 2002;50:402-4.

32. Fogt $\mathbf{F}$, Brien T, Brown CA, et al. Genetic alterations in serrated adenomas: comparison to conventional adenomas and hyperplastic polyps. Hum Pathol 2002;33:87-91.

33. Sawyer EJ, Cerar A, Hanby AM, et al. Molecular characteristics of serrated adenomas of the colorectum. Gut 2002;51:200-6.

34. Fu X, Li J, Li K, et al. Hypermethylation of APC promoter 1A is associated with moderate activation of Wnt signalling pathway in a subset of colorectal serrated adenomas. Histopathology 2009;55:554-63.

35. Miyoshi $\mathbf{Y}$, Ando $\mathrm{H}$, Nagase $\mathrm{H}$, et al. Germ-line mutations of the APC gene in 53 familial adenomatous polyposis patients. Proc Natl Acad Sci U S A 1992;89:4452-6.

36. Su LK, Barnes CJ, Yao W, et al. Inactivation of germline mutant APC alleles by attenuated somatic mutations: a molecular genetic mechanism for attenuated familial adenomatous polyposis. Am J Hum Genet 2000;67:582-90.

37. Friedl W, Meuschel S, Caspari R, et al. Attenuated familial adenomatous polyposis due to a mutation in the $3^{\prime}$ part of the APC gene. A clue for understanding the function of the APC protein. Hum Genet 1996;97:579-84.

38. Oh K, Redston M, Odze RD. Support for hMLH1 and MGMT silencing as a mechanism of tumorigenesis in the hyperplastic-adenoma-carcinoma (serrated) carcinogenic pathway in the colon. Hum Pathol 2005;36:101-11.

39. Yachida S, Mudali S, Martin SA, et al. Beta-catenin nuclear labeling is a common feature of sessile serrated adenomas and correlates with early neoplastic progression after BRAF activation. Am J Surg Pathol 2009;33:1823-32.

40. Fu X, Yang X, Chen K, et al. Retained cell-cell adhesion in serrated neoplastic pathway as opposed to conventional colorectal adenomas. J Histochem Cytochem 2011:59:158-66.

41. Wu JM, Montgomery EA, lacobuzio-Donahue CA. Frequent beta-catenin nuclear labeling in sessile serrated polyps of the colorectum with neoplastic potential. $A m \mathrm{~J}$ Clin Pathol 2008:129:416-23.

42. Sandmeier D, Benhattar J, Martin P, et al. Serrated polyps of the large intestine: a molecular study comparing sessile serrated adenomas and hyperplastic polyps. Histopathology 2009;55:206-13.

43. Joo M, Shahsafaei A, Odze RD. Paneth cell differentiation in colonic epithelial neoplasms: evidence for the role of the Apc/beta-catenin/Icf pathway. Hum Patho 2009:40:872-80

44. Fujita K, Yamamoto H, Matsumoto $T$, et al. Sessile serrated adenoma with early neoplastic progression: a clinicopathologic and molecular study. Am J Surg Pathol 2011; 35:295-304.

45. Aust DE, Baretton GB. Serrated polyps of the colon and rectum (hyperplastic polyps, sessile serrated adenomas, traditional serrated adenomas, and mixed polyps)proposal for diagnostic criteria. Virchows Arch 2010;457:291-7.

46. Hawkins NJ, Ward RL. Sporadic colorectal cancers with microsatellite instability and their possible origin in hyperplastic polyps and serrated adenomas. J Natl Cancer Inst 2001;93:1307-13.

47. Jass JR, Biden KG, Cummings MC, et al. Characterisation of a subtype of colorectal cancer combining features of the suppressor and mild mutator pathways. J Clin Pathol 1999;52:455-60.

48. Mirabelli-Primdahl L, Gryfe R, Kim H, et al. Beta-catenin mutations are specific for colorectal carcinomas with microsatellite instability but occur in endometrial carcinomas irrespective of mutator pathway. Cancer Res 1999;59:3346-51.

49. Morin PJ, Sparks AB, Korinek V, et al. Activation of beta-catenin-Tcf signaling in colon cancer by mutations in beta-catenin or APC. Science 1997:275:1787-90.

50. Esteller M, Sparks A, Toyota M, et al. Analysis of adenomatous polyposis coli promoter hypermethylation in human cancer. Cancer Res 2000;60:4366-71.

51. Nathke IS. The adenomatous polyposis coli protein: the Achilles heel of the gut epithelium. Annu Rev Cell Dev Biol 2004;20:337-66. 
52. Chen J, Rocken C, Lofton-Day C, et al. Molecular analysis of APC promoter methylation and protein expression in colorectal cancer metastasis. Carcinogenesis 2005:26:37-43

53. Suzuki $\mathbf{H}$, Watkins DN, Jair KW, et al. Epigenetic inactivation of SFRP genes allows constitutive WNT signaling in colorectal cancer. Nat Genet 2004;36:417-22.

54. O'Brien MJ, Yang S, Mack C, et al. Comparison of microsatellite instability, $\mathrm{CpG}$ island methylation phenotype, BRAF and KRAS status in serrated polyps and traditional adenomas indicates separate pathways to distinct colorectal carcinoma end points. Am J Surg Pathol 2006:30:1491-501.

55. Park SJ, Rashid A, Lee JH, et al. Frequent $\mathrm{CpG}$ island methylation in serrated adenomas of the colorectum. Am J Pathol 2003;162:815-22.
56. Dong SM, Lee EJ, Jeon ES, et al. Progressive methylation during the serrated neoplasia pathway of the colorectum. Mod Pathol 2005;18:170-8.

57. Dhir M, Yachida S, Van Neste L, et al. Sessile serrated adenomas and classical adenomas: an epigenetic perspective on premalignant neoplastic lesions of the gastrointestinal tract. Int J Cancer. Published Online First: 10 December 2010.

58. Fukuyama R, Niculaita R, Ng KP, et al. Mutated in colorectal cancer, a putative tumor suppressor for serrated colorectal cancer, selectively represses beta-catenindependent transcription. Oncogene 2008;27:6044-55.

59. Kohonen-Corish M, Sigglekow N, Susanto J, et al. Promoter methylation of the mutated in colorectal cancer gene is a frequent early event in colorectal cancer. Oncogene 2007;26:4435-41. 